\title{
Microstructure et profil des raies de diffraction des rayons $X$
}

D. Louër

Laboratoire de Chimie du Solide et Inorganique Moléculaire, UMR 6511 du CNRS, Institut de Chimie, Université de Rennes, avenue du Général Leclerc, 35042 Rennes cedex, France

\section{1- Introduction}

\section{2- Caractéristiques du profil des raies et des diagrammes de diffraction}

2.1 Origine du profil des raies de diffraction et convolution

2.2 Paramètres fonqamentaux du profil des raies de diffraction

2.3 Modélisation du profil des raies de diffraction

\section{3- Fondements théoriques}

3.1 Diffraction par des cristallites imparfaits

3.2 Taille apparente et forme des cristallites

3.2.1 Sphère

3.2.2 Cylindre

3.3 Microdistorsions anisotropes et dislocations

3.4 Fautes d'empilement

\section{4- La correction instrumentale}

4.1 La fonction instrumentale $g(x)$

4.2 Correction par déconvolution

4.3 Correction instrumentale des largeurs intégrales

\section{5- Approche simplifiée de l'analyse microstructurale}

5.1 Le diagramme de Williamson-Hall

5.2 Evaluation approximative des propriétés microstructurales

5.3 Diagramme de Williamson-Hall modifié

\section{6- Applications}

6.1 Cristallites isotropes sans distorsion : $\mathrm{CeO}_{2}$ ex-sulfate

6.2 Cristallites anisotropes sans distorsion : $\mathrm{ZnO}$ ex-hydroxynitrate

6.3 Cristallites anisotropes avec des fautes : $\mathrm{ZnO}$ ex-oxalate

6.4 Cristallites anisotropes avec des distorsions : $\mathrm{Ni}(\mathrm{OH})_{2}$

6.5 Cristallites isotropes avec des dislocations : $\mathrm{CeO}_{2}$ ex ammonium-nitrate

\section{7- Remarques finales}




\section{INTRODUCTION}

Différents types de défauts, généralement rassemblés sous le terme microstructure, contribuent à l'élargissement de la distribution des intensités dans une réflexion de Bragg. Les imperfections structurales rencontrées le plus souvent dans les matériaux réels concernent les distorsions réticulaires, la petite taille des cristallites et les fautes d'empilement. Dans le réseau d'un cristal parfait la position d'une maille élémentaire, repérée par les trois entiers $n_{1} n_{2} n_{3}$, est définie à partir de l'origine du cristal par le vecteur $\mathbf{R}_{n}=n_{1} \mathbf{a}_{1}+n_{2} \mathbf{a}_{2}+n_{3} \mathbf{a}_{3}$, où $\mathbf{a}_{1} \mathbf{a}_{2} \mathbf{a}_{3}$ sont les trois vecteurs de la maille unitaire. Dans le cas d'un solide distordu la position de l'origine de cette $n^{\text {ième }}$ maille est repérée par le vecteur $\mathbf{R}_{n}=n_{1} \mathbf{a}_{1}+n_{2} \mathbf{a}_{2}+n_{3} \mathbf{a}_{3}+\delta\left(n_{1} n_{2} n_{3}\right)$, où $\delta$ représente le déplacement de la maille induit par la présence des distorsions au sein du domaine cristallin. Ces distorsions se traduisent par une variation des distances réticulaires $d_{h k l}$ à l'intérieur d'un domaine de diffraction cohérente, appelé cristallite dans la suite de cet article. Cette variation, exprimée au travers d'une déformation relative $\Delta d l d$, peut provenir de la présence de dislocations, de lacunes pour des composés non-stœchiométriques, de fluctuations de distances inter-feuillets dans des solides lamellaires ou, encore, d'un gradient en composition. L'élargissement provoqué par cet effet de microcontrainte croît avec l'ordre des réflexions. Par ailleurs, lorsque les dimensions des cristallites deviennent inférieures à environ un millier de longueurs d'onde, le profil des raies de diffraction s'élargit indépendamment de l'ordre de la réflexion; c'est l'effet de taille. Enfin, la présence de fautes d'empilement dans les structures compactes contribue également à un accroissement de la largeur des distributions d'intensité, selon des règles qui dépendent pour chaque réflexion de combinaisons spécifiques des indices de Miller.

Dans le cas de poudres microcristallines, seule une variation unidimensionnelle de l'intensité diffractée avec l'angle de diffraction est disponible. C'est donc à partir du profil des raies $h k l$ contenues dans le diagramme qu'une information quantitative sur la microstructure du matériau peut être obtenue. Selon le nombre de réflexions disponibles, la caractérisation sera plus ou moins complète. Elle peut parfois, dans des cas favorables, conduire à une description tridimensionnelle des propriétés microstructurales. Pour cela, les techniques de modélisation du profil des raies de diffraction contribuent à repousser les limites d'application des méthodes d'analyse.

Cet article présente quelques aspects de la caractérisation microstructurale, à l'échelle nanométrique, à partir de l'analyse du profil des raies de diffraction des rayons $\mathrm{X}$. Si les théories principales sur les imperfections structurales étaient déjà bien établies vers les années cinquante, le renouveau de la diffraction par les poudres [1] a conduit à un regain d'intérêt pour l'analyse microstructurale dans les années récentes. Celui-ci s'est, en particulier, traduit par l'édition récente de l'ouvrage Defect and microstructure analysis by diffraction [2].

\section{CARACTERISTIQUES DU PROFIL DES RAIES ET DES DIAGRAMMES DE DIFFRACTION}

\subsection{Origine du profil des raies de diffraction et convolution}

Chaque profil de raie de diffraction observée dans un diagramme de poudre, $h(x)$, résulte d'une opération de convolution entre le profil de raie intrinsèque à l'échantillon, $f(x)$, et les effets instrumentaux, $g(x)$, selon l'équation:

$$
h(x)=\int f(y) g(x-y) \mathrm{d} y=f(x) * g(x),
$$

où $x$ et $y$ sont généralement exprimés en degrés $2 \theta$. Le profil de raie $f(x)$, représentatif de la microstructure du matériau, peut être lui-même la convolution de profils associés à différents 
types de défauts, à savoir les effets de taille $f^{T}(x)$, de distorsion $f^{D}(x)$ et de fautes d'empilement $f^{E}(x)\left[f(x)=f^{\prime}(x) * f^{D}(x) * f^{E}(x)\right]$. Quant à la fonction instrumentale $g(x)$ elle résulte de la convolution des diverses aberrations physiques et géométriques du diffractomètre employé, incluant la dispersion du domaine spectral utilisé pour l'enregistrement des données. Cette fonction instrumentale sera commentée dans la section 4.1 .

\subsection{Paramètres fondamentaux du profil des raies de diffraction}

Plusieurs paramètres mathématiques sont utilisés pour caractériser un profil de raie de diffraction des rayons $\mathrm{X}$. La position de la réflexion est souvent définie par l'angle $2 \theta_{0}$ pour lequel l'intensité de la réflexion est maximum. Il y a plusieurs mesures possibles de la dispersion angulaire de l'intensité. La largeur à mi-hauteur, FWHM (Full Width at Half Maximum), est la distance angulaire entre les deux angles pour lesquels l'intensité a une valeur moitié de sa valeur à la position du pic $\left(I_{0}\right)$. La largeur intégrale $\beta$ a des avantages théoriques considérables. Elle est définie comme l'intensité intégrale $(I)$ du profil de raie divisée par son intensité au maximum:

$$
\beta=\int I(2 \theta) \mathrm{d}(2 \theta) / I_{0}
$$

Elle est habituellement mesurée en degrés 20 à partir des données expérimentales, mais pour une analyse approfondie il est préférable d'utiliser $\beta^{*}\left[=\left(\beta_{2 \theta} \cos \theta\right) / \lambda\right]$ exprimée en unités réciproques $\left(\AA^{-1}\right)$.

Dans le contexte de l'analyse de Fourier, le profil des raies de diffraction est représenté par une série de Fourier, telle que:

$$
f(x)=\sum_{-\infty}^{+\infty}\left[\mathrm{A}_{n} \cos (2 \pi n x)+\mathrm{B}_{n} \sin (2 \pi n x)\right]
$$

où $x$ est la variable de l'espace réciproque et $n$ est le nombre harmonique de Fourier. En pratique, $x$ prend la forme de $\left(s-s_{0}\right) / \Delta s$, où $s=2 \sin \theta / \lambda, s_{0}=2 \sin \theta_{0} / \lambda, \Delta s=\left(2 \sin \theta_{2}-2 \sin \theta_{1}\right) / \lambda$, $\left(\theta_{1}, \theta_{2}\right)$ étant le domaine angulaire contenant toute l'intensité de la raie de diffraction et $\theta_{0}$ est l'angle de Bragg. Bien qu'ils ne soient pas une mesure de la largeur des raies, au sens strict du terme, les coefficients de Fourier sont physiquement reliés à la forme des raies de diffraction.

Enfin, la forme du profil des raies est définie par $\phi=F W H M / \beta$. Lorsque des fonctions analytiques sont utilisées, des facteurs de forme spécifiques leur sont alors affectés (voir section 2.3).

\subsection{Modélisation du profil des raies de diffraction}

L'ajustement d'un modèle analytique aux données expérimentales de diffraction par la poudre est couramment utilisé, notamment en présence de recouvrement des raies de diffraction. Dans la méthode de décomposition aucune information sur la structure atomique du matériau (hormis, éventuellement, les paramètres de maille et les conditions d'extinction sur $h k l$ ) n'est utilisée. Par contre, la méthode de Rietveld inclut l'affinement des coordonnées atomiques des éléments constituant le matériau [3]. La première approche est utilisée pour restituer les composantes élémentaires de Bragg et leurs caractéristiques. Quant à la méthode de Rietveld, une application secondaire de l'affinement des coordonnées atomiques peut être l'interprétation en termes physiques de l'élargissement des réflexions de Bragg du diagramme $[4,5]$. Dans la méthode de décomposition, l'intensité calculée $y\left(x_{i}\right)$ au point $x_{i}$ est exprimée en fonction de l'intensité intégrale $I_{k}$ des réflexions contenues dans le diagramme et d'une fonction 
normalisée $\Phi(x)$ utilisée pour représenter chaque profil de raie individuel $k$ :

$$
y_{\mathrm{cal}}\left(x_{i}\right)=\sum_{k} I_{k} \Phi\left(x_{i}-x_{k}\right)+b\left(x_{i}\right)
$$

où $b\left(x_{i}\right)$ est l'intensité du fond continu au point $x_{i}$. La sommation porte sur toutes les réflexions $k$ contribuant à l'intensité en $x_{i}$. Dans la méthode de Rietveld, l'intensité $I_{k}$ de chaque réflexion est remplacée par sa signification physique en terme structural, c'est-à-dire:

$$
I_{k}=\mathrm{m}_{k}(\mathrm{Lp})_{k}\left|\mathrm{~F}_{k}\right|^{2} \mathrm{P}_{k}
$$

où $\mathrm{m}_{k}$ est le facteur de multiplicité, $\mathrm{Lp}$ le facteur de Lorentz-polarisation, $\mathbf{P}_{k}$ le facteur d'orientation préférentielle et $\mathrm{F}_{k}$ le facteur de structure de la réflexion $k$. La dépendance angulaire de la largeur $F W H M$ des raies de diffraction est habituellement décrite par l'expression quadratique suivante:

$$
F W H M^{2}=\mathrm{Utg}^{2} \theta+\mathrm{Vtg} \theta+\mathrm{W}
$$

Pour les deux approches, la méthode des moindres-carrés est alors généralement utilisée pour estimer la valeur des paramètres ajustables du modèle à partir des données expérimentales (quantité minimisée $\Sigma\left[y_{\text {obs }}(x)-y_{\text {cal }}(x)\right]^{2}$ ). La validité du modèle est appréciée au travers de facteurs d'accord variés (voir réf. 1).

Les fonctions $\Phi$ (pour une définition mathématique précise, voir références 1 et 3 ) les plus communément utilisées sont dérivées des fonctions de Gauss $(G)$ et de Cauchy $(C)$. La fonction de pseudo-Voigt (p-V) est la somme de composantes $G$ et $C[\eta C+(1-\eta) G)]$ dans une proportion mesurée par le facteur de forme $\eta(\eta=1$ pour $C$ et 0 pour $G)$. La fonction de Pearson VII est [ $\left.C^{m}\right]$ où l'exposant $m$ est le facteur de forme ( $m=1$ pour $C$ et $m=\infty$ pour $G$ ). La fonction de Voigt est la convolution de composantes $C$ et $G[C * G]$. Les limites d'applicabilité de cette dernière correspondent aux fonctions de Cauchy et de Gauss. Elles se traduisent par des valeurs particulières du paramètre de forme $\phi$, à savoir 0,6366 pour la limite de Cauchy et 0,9394 pour celle de Gauss. Contrairement à la fonction de Voigt, les fonctions de Pearson VII et pseudo-Voigt peuvent, dans une certaine mesure, être utilisées lorsque des raies de diffraction sortent de ces limites, par exemple lorsque $m<1$ ou $\eta>1$. Une telle situation correspond à une forme qualifiée de super-Lorentzienne. En pratique, lors des modélisations il est difficile d'éviter les erreurs de troncature. Ainsi, pour des fonctions de Cauchy, $99 \%$ de l'aire du profil est prise en compte lorsque le domaine angulaire est environ 63 fois la largeur $F W H M$ [6]. En plus de cet effet de troncature on doit également mentionner la difficulté de l'estimation du fond continu dans un diagramme de diffraction lorsque les raies sont larges et/ou la densité des réflexions élevée. Ces deux facteurs sont sources de propagation d'erreurs qui doivent être minimisées dans toute analyse.

La fonction de pseudo-Voigt décrit souvent de manière correcte le profil des raies de diffraction d'un diagramme de poudre; toutefois, le facteur de forme $\eta$ ne peut pas être interprété en termes de propriété physique. Il a été proposé d'utiliser les composantes de Gauss et de Cauchy de cette fonction pour dériver des paramètres microstructuraux isotropes de taille et de distorsion [7]. Pour cela la dépendance angulaire de la composante de Gauss $F W H M_{G}$ est décrite par une équation similaire à (6) et celle de Cauchy par:

$$
F W H M_{C}=\mathrm{X} \operatorname{tg} \theta+\mathrm{Y} / \cos \theta .
$$

Ces expressions (6) et (7) renferment des termes variant en $\cos ^{-1} \theta$ et en $\operatorname{tg} \theta$, lesquels peuvent faire l'objet d'une interprétation physique en termes, respectivement, de taille apparente 
moyenne des cristallites, par analogie avec l'équation de Scherrer (voir équ. 11 ci-dessous, lorsque la largeur, $\beta$ ou $F W H M$, est exprimée en ${ }^{\circ} 2 \theta$ ), et de distorsion (voir équ. 15 cidessous). Ces approches sont utilisées dans de nombreux programmes de la méthode de Rietveld et des extensions aux cas d'élargissements appelés anisotropes sont proposées [8].

\section{FONDEMENTS THEORIQUES}

\subsection{Diffraction par des cristallites imparfaits}

Le profil de raie $f(s)$ associé à des cristallites de petite taille et à des imperfections structurales est représenté $[9,10]$, à une constante près, par:

$$
f(s)=\int V(t) y(t) \exp (-2 \pi \mathrm{i} s t) \mathrm{d} t
$$

où $V(t)$ est la contribution associée à la taille du cristallite et $y(t)$ est la valeur moyenne dans l'ensemble du cristallite du produit $F F^{*}$ des facteurs de structure complexes de paires de mailles séparées d'une distance $t$ dans la direction perpendiculaire aux plans diffractants. Le profil de raie, exprimé en fonction de la variable réciproque $s$, est alors proportionnel à la transformée de Fourier de $V(t) y(t)$.

$V(t)$ est interprété comme étant le volume commun à un cristallite et à son 'double' obtenu par une translation $t$ parallèlement au vecteur diffraction. Si les distorsions de réseau sont négligeables, $y(t)$ est une constante, et l'inverse de la pente initiale de la transformée de Fourier du profil de raie normalisé correspond à la taille apparente de Fourier [11]:

$$
\varepsilon_{\mathrm{F}}=-V(0) / V(t)_{t=0}
$$

où $V(0)$ est le volume moyen du cristallite. $\varepsilon_{\mathrm{F}}$ est une valeur moyenne de l'épaisseur du cristallite dans la direction du vecteur diffraction. De plus, la dérivée seconde $V^{\prime \prime}(t)$ est proportionnelle à la distribution des épaisseurs, mesurées dans la direction du vecteur diffraction [11].

La largeur intégrale $\beta^{T}$, due à l'effet de taille, est reliée à la fonction volume $V(t)$, au travers de l'équation:

$$
\beta^{\mathrm{T}} *=V(0) / \int V(t) \mathrm{d} t
$$

L'inverse de cette quantité est une moyenne volumétrique des épaisseurs du cristallite, mesurée dans une direction perpendiculaire aux plans diffractants. C'est la taille apparente $\varepsilon_{\beta}$ :

$$
\varepsilon_{\beta}=1 / \beta^{\mathrm{T}} \text {, }
$$

connue sous le nom de relation de Scherrer.

L'interprétation de $y(t)$ n'est pas aussi simple, car elle dépend de la nature des imperfections structurales associées au vecteur $\delta$ défini dans l'introduction. Dans l'approche de Fourier la composante des distorsions $\mathrm{e}_{n}$ est exprimée par le rapport $\Delta \mathrm{L} / \mathrm{L}$, où $\mathrm{L}$ ( $=n d_{h k l}$ ou $n / \Delta s$ selon la période $d^{*}$ ou $\Delta s$ sur laquelle le nombre harmonique de Fourier $n$ est calculé [12]) est la distance en l'absence de distorsion entre paires de mailles séparées par $n$ mailles et $\Delta \mathrm{L}$ est la variation de distance due à la présence de distorsions. Dans l'approche de WarrenAverbach-Bertaut [12], où les séries de Fourier sont préférées aux transformées de Fourier, les coefficients complexes de Fourier $\mathrm{C}_{\mathrm{n}}(l)\left[=\mathrm{A}_{n}(l)+\mathrm{iB}_{n}(l)\right]$ pour une réflexion d'ordre $l$ sont exprimés par le produit du coefficient de taille $\mathrm{A}_{n}^{\mathrm{T}}$ réel, indépendant de l'ordre $l$, et du coefficient de distorsion complexe $\mathrm{C}_{n}{ }^{\mathrm{D}}(l)\left[=\mathrm{A}_{n}{ }^{\mathrm{D}}(l)+\mathrm{iB}_{n}{ }^{\mathrm{D}}(l)\right]$, fonction de l'ordre $l$ de la 
réflexion. Les deux coefficients peuvent être séparés si deux ordres, au moins, sont disponibles. Ceci peut se faire à l'aide des graphes $\ln \mathrm{A}_{n}(l)$ en fonction de $l^{2}$, fondés sur les développements limités d'un cosinus et d'un logarithme:

$$
\ln \mathrm{A}_{n}(l)=\ln \mathrm{A}_{n}{ }^{\mathrm{T}}-2 \pi^{2} l^{2} n^{2}<\mathrm{e}_{n}{ }^{2}>
$$

Le développement limité du logarithme peut être évité au moyen d'une version linéaire de l'analyse [13]:

$$
\mathrm{A}_{n}(l)=\mathrm{A}_{n}^{\mathrm{T}}-\mathrm{A}_{n}^{\mathrm{T}} 2 \pi^{2} l^{2} n^{2}<\mathrm{e}_{n}^{2}>
$$

Dans ces expressions, $\left\langle\mathrm{e}_{n}{ }^{2}\right\rangle$ est la moyenne quadratique des distorsions, souvent appelée microcontrainte. Dans cette approche de Fourier, l'inverse de la pente à l'origine de $A_{n}=f(L)$ est égal à la taille apparente des cristallites $\varepsilon_{\mathrm{F}}$, qui est définie comme une taille du cristallite pondérée en surface, dans une direction perpendiculaire aux plans $h k l$ considérés, c'est-à-dire selon l'équation (9):

$$
\left(\frac{\mathrm{dA}_{\mathrm{L}}^{\mathrm{T}}}{\mathrm{dL}}\right)_{\mathrm{L}=0}=-\frac{1}{\varepsilon_{\mathrm{F}}} .
$$

La distribution de tailles $V^{\prime \prime}(t)$, mentionnée plus haut et plus justement appelée distribution des longueurs de colonnes de mailles, est aussi accessible au travers de la dérivée seconde de la fonction $\mathrm{A}_{n}{ }^{\mathrm{T}}=\mathrm{f}(\mathrm{L})$.

Conformément à l'équation (10), les coefficients de Fourier permettent une évaluation de la largeur intégrale $\beta^{*}\left(=\Delta s / \Sigma_{n}\left|C_{n}\right|\right)$. Ce calcul ne fait aucune hypothèse sur la forme des raies de diffraction. Wagner [14] a montré qu'il était souvent préférable d'utiliser les coefficients $|C(n)|$ au lieu de $\mathrm{A}(n)$ dans les calculs numériques, afin d'éviter des erreurs liées au choix de l'origine de la série de Fourier. En ce qui concerne la largeur intégrale une microcontrainte apparente $\eta_{\beta}$, définie à partir de la composante $\beta^{\mathrm{D} *}$, relative à l'effet de distorsion, a été introduite par Stokes et Wilson [15]:

$$
\eta_{\beta}=2 \beta^{\mathrm{D} *} / d^{*}=\beta_{2 \theta}^{\mathrm{D}} \operatorname{cotg} \theta
$$

et discutée ultérieurement $[16,17]$. Deux modèles simples de contraintes pour lesquels $\eta_{\beta}$ est relié à la distorsion vraie ont été proposés [15-17]. Dans le premier modèle l'évaluation de la composante de distorsion vraie, notée ê $(=\Delta d / d)$, repose sur l'hypothèse que l'équation de Bragg est valide sur tout le domaine angulaire correspondant à $d \pm \Delta d$. Dans ces conditions:

$$
\hat{\mathrm{e}}=\eta_{\beta} / 4 \text {. }
$$

Dans le second modèle, la composante de microcontrainte est définie par la valeur quadratique moyenne de la distorsion, notée ẽ $\left(=\left\langle\mathrm{e}^{2}\right\rangle^{1 / 2}\right)$, et s'exprime comme suit:

$$
\tilde{\mathrm{e}}=\eta_{\beta} /\left(2(2 \pi)^{1 / 2}\right) \cong \eta_{\beta} / 5
$$

Dans le cas le plus général, ces deux propriétés microstructurales (taille et distorsions) sont le plus souvent anisotropes, ce qui implique un élargissement des raies de diffraction directement dépendant de la direction du vecteur diffraction et, donc, des indices $h k l$ des réflexions. 


\subsection{Taille apparente et forme des cristallites}

Seules des tailles apparentes dans des directions cristallographiques privilégiées peuvent être dérivées de l'analyse du profil des raies de diffraction. En pratique, il est clair que les dimensions réelles (et la forme) des cristallites présentent un intérêt tout particulier. En principe, elles peuvent être obtenues à partir de $\varepsilon_{\beta}$ et $\varepsilon_{F}$ pour des cristallites possédant une morphologie bien définie, comme par exemple une sphère [9], un parallélépipède [16], un cylindre [18] ou un prisme droit à base hexagonale [19]. En pratique, la situation est souvent compliquée par la superposition exacte de réflexions, due à la symétrie cristalline du matériau (multiplicité). Le profil de raie observé est alors moyenné par la sommation de composantes de Bragg élémentaires présentant des élargissements différents. Il s'agit là d'un effet qui, combiné à une distribution de taille des cristallites, limite considérablement les possibilités d'extraire la morphologie précise des cristallites. Toutefois, deux cas présentent un intérêt particulier pour les exemples présentés ci-dessous: le modèle isotrope sphérique et le modèle cylindrique dont l'axe principal est colinéaire avec l'axe sénaire de la symétrie hexagonale. Notons en passant que ce dernier modèle peut être utile pour décrire des cristallites dont la forme varie d'un disque à une aiguille.

\subsubsection{Sphère}

Pour des cristallites sphériques de diamètre $\mathrm{D}$, la taille apparente est constante, quelle que soit la direction du vecteur de diffraction. Le diamètre peut être obtenu à partir de la largeur intégrale, $D_{\beta}\left(=4 \varepsilon_{\beta} / 3\right)$, et de l'approche de Fourier, $D_{F}\left(=3 \varepsilon_{F} / 2\right)$. Puisque $D_{\beta}=D_{F}$ pour un seul cristallite, le rapport idéal des tailles apparentes $\varepsilon_{\beta} / \varepsilon_{\mathrm{F}}$ est 1.125 . L'obtention de valeurs différentes implique la présence d'une distribution de taille des cristallites. L'effet d'une distribution de tailles sur le profil de raies de diffraction a été discuté en détail récemment [20].

\subsubsection{Cylindre}

Pour un cylindre de diamètre $D$ et hauteur $H$, les relations entre les tailles apparentes $\left(\varepsilon_{\beta}\right.$ et $\left.\varepsilon_{F}\right)$ et $\mathrm{D}$ et $\mathrm{H}$ dépendent, d'une part, de l'angle $\phi_{z}$ définissant l'orientation du vecteur diffraction par rapport à l'axe du cylindre (ou $c^{*}$ ) et, d'autre part, de l'angle diagonal $\Phi[\operatorname{tg} \Phi=\mathrm{D} / \mathrm{H}] \mathrm{du}$ modèle. D'après Langford et Louër (équ. 10,17 et 18 dans la réf. 18), les relations correspondantes sont:

$$
\begin{aligned}
& \left.\varepsilon_{\beta}=(D / \pi) \csc _{z}\left[8 / 3+2 q \cos ^{-1} q-(1 / 2 q) \sin ^{-1} q-(5 / 2)\left(1-q^{2}\right)^{1 / 2}+(1 / 3)\left(1-q^{2}\right)^{3 / 2}\right)\right], 0 \leq \phi_{z} \leq \Phi \\
& \varepsilon_{\beta}=D \csc \phi_{z}[8 /(3 \pi)-(1 / 4 q)], \Phi \leq \phi_{z} \leq \pi / 2, \\
& \text { où } q=H\left(\operatorname{tg} \phi_{z}\right) / D, \text { et } \\
& \varepsilon_{F}=\left[\left(\cos \phi_{z} / H\right)+\left(4 \sin \phi_{z} / \pi D\right)\right]^{-1} .
\end{aligned}
$$

Le diamètre $\mathrm{D}$ et la hauteur $\mathrm{H}$ se déduisent respectivement des réflexions $h 00$ ou $h k 0\left(\phi_{\mathrm{z}}=\right.$ $90^{\circ}$ ) et $00 l\left(\phi_{z}=0^{\circ}\right)$. Ces dernières équations peuvent être mises en œuvre dans une approche de type moindres-carrés pour calculer hauteur et diamètre moyens de cristallites, dans la mesure où les dimensions apparentes sont connues pour diverses directions cristallographiques $[21,22]$. Elles furent également utilisées dans la modélisation d'un diagramme complet de diffraction des rayons $\mathrm{X}$ [23]. 


\subsection{Microdistorsions anisotropes et dislocations}

En présence de dislocations, la dépendance des microdistorsions $\left\langle\mathrm{e}_{n}{ }^{2}\right\rangle$ avec $h k l$ peut être exprimée à l'aide d'un facteur de contraste, C. Puisque les dislocations sont des défauts linéaires, leur contraste dépend fortement des directions relatives du vecteur de Burgers de la dislocation et du vecteur de diffraction. Cet effet est bien connu dans le domaine de la microscopie électronique à transmission (MET). D'après Krivoglaz [24] et Wilkens [25], pour de faibles valeurs de $\mathrm{L}$, le terme de microcontrainte introduit dans l'approche de WarrenAverbach-Bertaut (équ. 12) peut être exprimé en fonction des paramètres de dislocations, à savoir la densité de dislocation $(\rho)$, le module du vecteur de Burgers (b) et le rayon effectif de coupure des dislocations $\left(R_{\mathrm{e}}\right)$ :

$$
<\mathrm{e}_{n}^{2}>=\left(\rho \mathrm{Cb}^{2} / 4 \pi\right) \ln \left(R_{\mathrm{e}} / \mathrm{L}\right) .
$$

L'anisotropie est introduite par le facteur de contraste $C$, qui doit être calculé pour chaque raie analysée. Ces calculs sont rapportés pour des matériaux cubiques [26] et hexagonaux [27]. Pour des cristaux de symétrie cubique, les facteurs moyens de contraste des dislocations peuvent être écrits de la façon suivante [26]:

$$
\mathrm{C}=\mathrm{C}_{h 00}\left(1-q H^{2}\right) \text {, }
$$

ou $\mathrm{C}_{h 00}$ est le facteur moyen de contraste pour les réflexions $h 00, q$ est un paramètre qui dépend des constantes élastiques du cristal et de la nature (vis ou coin) des dislocations, et

$$
H^{2}=\left(h^{2} k^{2}+h^{2} l^{2}+k^{2} l^{2}\right) /\left(h^{2}+k^{2}+l^{2}\right)^{2} .
$$

Les valeurs de $\mathrm{C}_{h 00}$ peuvent être obtenues à partir de tables rapportées dans la réf. 28. Plus particulièrement, $\mathrm{C}_{h 00}$ et $q$ ont été évalués pour des dislocations avec des vecteurs de Burgers $a / 2<110>$ et $a / 2<111>$. La densité de dislocation est alors extraite de l'équation (12) après insertion du facteur de contraste:

$$
\ln \mathrm{A}(\mathrm{L})=\ln \mathrm{A}^{\mathrm{T}}(\mathrm{L})-\left(\pi \mathrm{b}^{2} / 2\right) \rho \mathrm{L}^{2} \ln \left(R_{\Theta} / \mathrm{L}\right)\left(d^{* 2} \mathrm{C}\right)+O\left(d^{* 4} \mathrm{C}^{2}\right),
$$

où $O$ correspond aux termes en $d^{* 2} \mathrm{C}$ d'ordre supérieur, généralement non interprétés.

\subsection{Fautes d'empilement}

L'effet de fautes d'empilement sur les profils de raies de diffraction a été discuté en détail par Guinier [9], Wilson [10] et Warren [12]. Les domaines de diffraction cohérente sont alors limités par la présence des fautes. La largeur intégrale correspondante est alors inversement proportionnelle à la distance moyenne entre les plans $h k l$ concernés. Cet élargissement des raies de diffraction est analogue à l'effet de taille décrit plus haut. En effet, il n'y a pas de dépendance directe avec l'ordre de la réflexion mais, par contre, la dépendance existe avec les indices $h k l$. Dans le cas de matériaux à symétrie cubique, on distingue les effets de fautes de déformation de ceux de croissance ou de micromaclage. Les fautes d'empilement ont alors trois effets essentiels sur les profils de raies: la taille apparente est modifiée, les fautes de déformation provoquent le déplacement de certaines réflexions dans le cas d'une symétrie cubique et les fautes par maclage entraînent des asymétries. La situation la plus simple se rencontre pour des matériaux de symétrie hexagonale, avec des fautes d'empilement affectant des plans perpendiculaires à la direction $c$. La séquence hexagonale compacte $\mathrm{ABA}$... est alors remplacée par la suite $A B A B A B_{\perp} C B C B C B \ldots$ où une faute s'est intercalée. Certaines réflexions s'élargissent alors de manière symétrique sans déplacement de leur position. Dans ce 
cas $[10,21]$, les largeurs intégrales $\beta^{\mathrm{E}}$ dues aux fautes répondent aux conditions suivantes:

$$
\begin{array}{lll}
\beta^{\mathrm{E}_{*}}=0 & \text { pour } & h-k=3 n \text { et } h k 0 \\
\beta^{\mathrm{E} *}=\alpha \cos \phi_{\mathrm{z}} / 2 c & \text { pour } & h-k=3 n \pm 1, l \text { impair, } \\
\beta^{\mathrm{E} *}=3 \alpha \cos \phi_{\mathrm{z}} / 2 c & \text { pour } & h-k=3 n \pm 1, l \text { pair, }
\end{array}
$$

où $\alpha$ est la probabilité de faute d'empilement et $\phi_{Z}$ l'angle entre le vecteur de diffraction et $c^{*}$.

\section{LA CORRECTION INSTRUMENTALE}

\subsection{La fonction instrumentale $g(x)$}

Le profil des raies de diffraction d'origine instrumentale, $g(x)$, résulte de la distribution de longueurs d'onde dans le faisceau incident convoluée avec les diverses fonctions décrivant les aberrations de l'instrument (dimensions finies de la source et de la fente réceptrice, planéité de l'échantillon, divergence axiale du faisceau, etc.) [29]. La largeur des raies de diffraction, dites instrumentales, ainsi que leur forme varient de manière continue avec l'angle $2 \theta$. Un exemple de variation angulaire de $F W H M_{g}$ (appelée Fonction de Résolution Instrumentale, FRI) et $\beta_{g}$, ainsi que des facteurs de forme des fonctions ajustées aux raies expérimentales, est présenté sur la figure 1, pour une géométrie de type Bragg-Brentano opérant en rayonnement monochromatique $\left(\mathrm{CuK \alpha _{1 }}\right)$ [30]. Ce type de caractéristiques instrumentales est généralement obtenu à partir de solides de références, supposés exempts de toute imperfection structurale et possédant des domaines de diffraction cohérente suffisamment grands pour ne pas provoquer d'élargissement des raies de diffraction. Plusieurs matériaux avec des coefficients linéaires d'absorption $\mu$ différents, calculés ici pour la radiation $\mathrm{CuK \alpha _{1 }}$, ont été proposés, tels que $\mathrm{BaF}_{2}$ $\left(\mu=1304 \mathrm{~cm}^{-1}\right), \mathrm{LaB}_{6}\left(\mu=1138 \mathrm{~cm}^{-1}\right), \mathrm{KCl}\left(\mu=258 \mathrm{~cm}^{-1}\right)$ et $\mathrm{Al}_{2} \mathrm{O}_{3}\left(\mu=127 \mathrm{~cm}^{-1}\right) . \mathrm{En}$ pratique, si la même substance chimique que le matériau étudié ne peut être utilisée, le choix du solide de référence se portera sur celui qui possède le coefficient d'absorption le plus voisin.

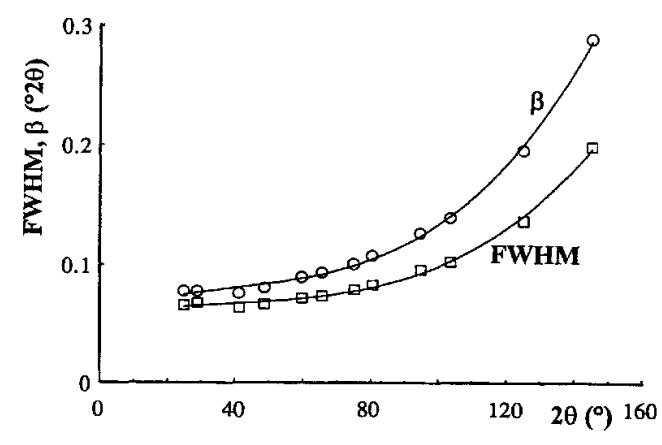

(a)

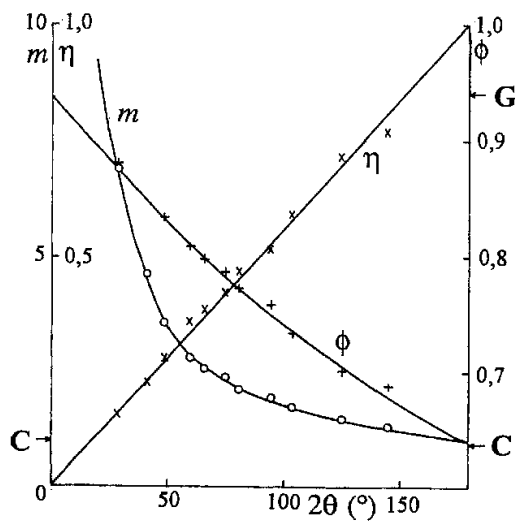

(b)

Figure 1: Caractéristiques de la fonction instrumentale $g(x)$ en géométrie Bragg-Brentano, radiation monochromatique $\mathrm{CuK \alpha} \alpha_{1}$, échantillon de référence $\mathrm{BaF}_{2}$ [30]. Variations angulaires (a) des largeurs expérimentales $F W H M$ et $\beta_{2 \theta}$, et $(b)$ des facteurs de forme $(m, \eta, \phi)$ des fonctions de Pearson VII, pseudo-Voigt et Voigt ajustées aux profils des raies de diffraction observés. 


\subsection{Correction par déconvolution}

Dans la mesure où le profil de raie de diffraction $f(x)$ complet est recherché ou que les coefficients de Fourier sont utilisés pour la caractérisation microstructurale, une opération de déconvolution doit être mise en œuvre. Une méthode pour obtenir le profil $f(x)$ (et/ou ses coefficients de Fourier) consiste à exprimer $h(x)$ et $g(x)$ sous forme de séries de Fourier, définies sur le même intervalle, et à utiliser la propriété multiplicative des transformées de Fourier de fonctions convoluées, c'est-à-dire:

$$
\mathrm{F}(t)=\mathrm{H}(t) / \mathrm{G}(t)
$$

où $\mathrm{F}(t), \mathrm{G}(t), \mathrm{H}(t)$ sont équivalents aux coefficients $\mathrm{F}(n), \mathrm{G}(n), \mathrm{H}(n)$ pour des valeurs discrètes de $t . f(x)$ peut alors être synthétisée par inversion de $\mathrm{F}(t)$. Cette approche, ou méthode de Stokes [31], est très largement utilisée. Elle présente toutefois quelques difficultés inhérentes à l'instabilité mathématique de l'opération de déconvolution [2]. Toutefois, lorsque ces difficultés sont négligeables $[h(x)$ est large par rapport à $g(x)]$ un avantage de la méthode de Stokes est de conduire directement à $\beta_{f}^{*}\left(=\Delta \mathrm{s} / \Sigma_{n} \mathrm{~A}_{n}\right)$ (voir équ. 10). Cette propriété prend tout son sens lorsque la méthode de Voigt décrite ci-après ne peut être appliquée (comme, par exemple, dans le cas de profils super-Lorentziens).

\subsection{Correction instrumentale des largeurs intégrales}

Si les profils de raie $h(x), f(x)$ et $g(x)$ sont supposés avoir la forme d'une fonction de Voigt, Langford $[32,16]$ a montré qu'il est possible de déterminer les composantes de Cauchy et de Gauss des profils de raies expérimentaux $h(x)$ et $g(x)$, à partir desquelles celles de $f(x)$ sont obtenues au travers des équations:

$$
\begin{aligned}
& \beta_{f C}=\beta_{h C}-\beta_{g C,}, \\
& \left(\beta_{f G}\right)^{2}=\left(\beta_{h G}\right)^{2}-\left(\beta_{g G}\right)^{2} .
\end{aligned}
$$

Les composantes $\beta_{f C}$ et $\beta_{f G}$ peuvent alors être combinées pour obtenir $\beta_{f}$. Cette méthode est précise, mais elle ne peut s'appliquer que lorsque les profils sont symétriques et que les facteurs de forme $\phi_{h}$ et $\phi_{g}$ sont compris entre les limites 0,6366 et 0,9394 .

\section{APPROCHE SIMPLIFIEE DE L'ANALYSE MICROSTRUCTURALE}

\subsection{Le diagramme de Williamson-Hall}

Le diagramme de Williamson-Hall [33] représente les variations de $\beta_{f}^{*}$ (ou $F W H M_{f}^{*}$ ) des réflexions $h k l$ en fonction de $d^{*}(=2 \sin \theta / \lambda)$. Il repose sur l'approximation de profils de raies ayant une forme de Cauchy et ne devrait donc être utilisé que qualitativement [le graphe $\beta_{f}^{*^{2}}=$ $\mathrm{f}\left(d^{*^{2}}\right)$ fondé sur une forme Gaussienne du profil des raies est également utilisé]. Il joue toutefois un rôle important car il donne un aperçu global des effets microstructuraux en fonction de la variable réciproque et des indices $h k l$ des réflexions. Il informe sur la nature des défauts à l'origine de l'élargissement (isotrope ou anisotrope) des raies de diffraction et permet donc d'orienter vers une analyse microstructurale ultérieure plus approfondie.

\subsection{Evaluation approximative des propriétés microstructurales}

Selon les hypothèses utilisées pour représenter le profil des raies relatif aux effets respectifs de taille et de microcontrainte des expressions approchées ont été dérivées, par exemple pour les 
combinaisons:

$$
\begin{array}{ll}
\text { Cauchy-Cauchy } & \beta_{f}^{*}=\varepsilon_{\beta}^{-1}+2 \mathrm{e} d^{*} \\
\text { Gauss-Gauss } & \beta_{f^{*}}=\left(\varepsilon_{\beta}{ }^{-1}\right)^{2}+4 \mathrm{e}^{2} d^{*^{2}} \\
\text { Cauchy-Gauss } & \beta_{f}^{*^{2}}=\left(\varepsilon_{\beta}{ }^{-1}\right) \beta_{f}^{*}+4 \mathrm{e}^{2} d^{*^{2}}
\end{array}
$$

où e est la composante réelle des microcontraintes, dont l'interprétation dépend du modèle de microcontrainte choisi [15,17], décrit par l'équation (16) ou (17). A partir du modèle CauchyGauss (équ. 29), fondé sur une approximation de la fonction de Voigt, Langford [16] propose l'usage du diagramme 'taille-contrainte moyen' $\left(\beta_{f}^{*} / d^{*}\right)^{2}$ en fonction de $\beta_{f}^{*} /\left(d^{*}\right)^{2}$ pour estimer les microcontraintes (ordonnée à l'origine) et taille (pente) dans le cas d'une microstructure à caractères isotropes. Dans cette interprétation la composante de Cauchy $\beta_{f c}{ }^{*}$ est affectée uniquement à l'effet de taille et celle de Gauss $\beta_{f G} *$ aux microcontraintes.

\subsection{Diagramme de Williamson-Hall modifié}

Lorsque l'anisotropie d'élargissement des raies de diffraction est due à la présence d'une densité de dislocations $\rho$, les largeurs $\mathrm{B}_{f}^{*}\left(\beta_{f}^{*}\right.$ et $\left.F W H M^{*}\right)$ peuvent être interprétées à partir de diagrammes de Williamson-Hall modifiés, tels que $[34,35]$ :

$$
\mathrm{B}_{f}^{*}=(1 / \varepsilon)+\left(\pi \mathrm{M}^{2} \mathrm{~b}^{2} / 2\right) \rho^{1 / 2}\left(d^{*} \mathrm{C}^{1 / 2}\right)^{2}+O\left(d^{*} \mathrm{C}^{1 / 2}\right)^{4},
$$

où $\varepsilon\left(\varepsilon_{\beta}\right.$ ou $\left.\varepsilon_{F W H M} / 0.9\right)$ est la taille apparente moyenne et $M$ une constante dépendant du rayon de coupure des dislocations (les autres termes sont définis dans la section 3.3).

\section{APPLICATIONS}

\subsection{Cristallites isotropes sans distorsion: $\mathrm{CeO}_{2}$ ex-sulfate}

Le diagramme de Williamson-Hall pour un échantillon d'oxyde de cérium obtenu par traitement thermique à $700^{\circ} \mathrm{C}$ de cérine hydratée, préparée elle-même à partir d'une solution de sulfate de cérium, a montré que les largeurs intégrales $\beta_{*^{*}}$ de 15 réflexions du diagramme, obtenues par l'analyse de Voigt, sont alignées sur une droite de pente nulle, à l'erreur expérimentale près [36]. Le matériau est donc exempt de distorsions réticulaires et les cristallites ont en moyenne une forme sphérique avec une dimension apparente moyenne $\left\langle\varepsilon_{\beta}\right\rangle$ estimée de $270(10) \AA$. Le diamètre moyen se déduit de la relation donnée dans la section 3.2.1, c'est-à-dire $\left\langle\mathrm{D}_{\beta}\right\rangle=360(13) \AA$.

L'analyse de Fourier a également été appliquée aux mêmes réflexions à l'aide du logiciel ProfFOU [37]. La figure 2 montre que les transformées de Fourier obtenues se superposent, ce qui confirme l'absence de distorsions et la forme sphérique des cristallites. La taille apparente moyenne de Fourier $\left\langle\varepsilon_{\mathrm{F}}\right\rangle$, déterminée à partir des pentes à l'origine dans les directions perpendiculaires aux plans $h k l$, est $180(12) \AA$. Le diamètre moyen $\left\langle\mathrm{D}_{\mathrm{F}}\right\rangle$ (voir section 3.2.1) calculé à partir de l'analyse de Fourier est 270(18) $\AA$.

La différence entre les diamètres $\left\langle\mathrm{D}_{\beta}\right\rangle$ et $\left\langle\mathrm{D}_{\mathrm{F}}>\right.$ traduit la présence d'une distribution de taille des cristallites. En effet, la valeur du rapport $\left\langle\varepsilon_{\beta}\right\rangle /\left\langle\varepsilon_{\mathrm{F}}\right\rangle$ est 1,5, alors que pour des cristallites de même taille elle est de 1,125. 


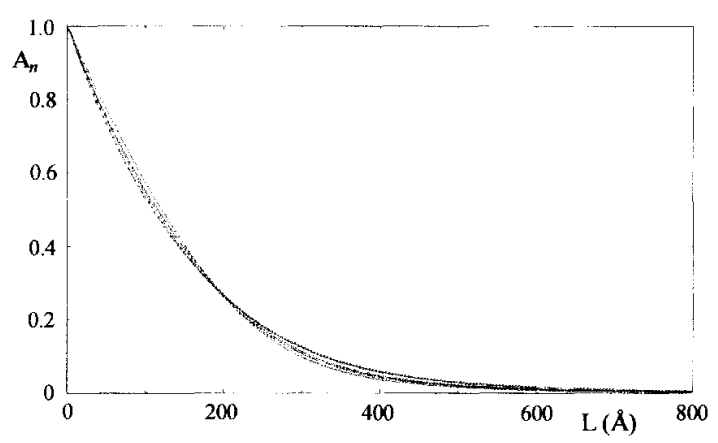

Figure 2: Coefficients de Fourier en cosinus $\mathrm{A}_{n}$ en fonction de $\mathrm{L}(\AA)$ pour quinze réflexions de $\mathrm{CeO}_{2}$ [36].

\subsection{Cristallites anisotropes sans distorsion: $\mathrm{ZnO}$ ex-hydroxynitrate}

Le diagramme de Williamson-Hall d'un échantillon de $\mathrm{ZnO}$, obtenu par décomposition thermique à $210^{\circ} \mathrm{C}$ de l'hydroxynitrate [38], montre que $\beta_{f}^{*}$ est similaire, à l'erreur expérimentale près, pour les ordres différents des réflexions $h 00, h 0 h$ et $00 l$ (figure $3 a$ ). Ceci traduit l'absence de distorsions mesurables dans l'échantillon. L'ordonnée à l'origine pour les réflexions $00 l$ est beaucoup plus faible que celle qui est observée pour les réflexions $h k 0$, ce qui est conforme avec une morphologie prismatique des cristallites caractérisée par une hauteur plus grande que le diamètre de la base. Le fait que la différence entre $\beta_{f}^{*}$ pour les raies $h 00$ et $h k 0$ soit faible, traduit la présence d'une section moyenne circulaire. Ces déductions sont confortées par le comportement de $\beta_{f}^{*}$ pour les réflexions $h 0 l$, pour lesquelles l'angle $\phi_{z}$ entre le vecteur de diffraction et le plan de base, croît de 0 à $\pi / 2$. Une analyse moindres carrés à l'aide des équations (18) a permis d'obtenir le modèle morphologique présenté sur la figure $3 b$ et caractérisé par une hauteur 270(60) $\AA$ et un diamètre 180(10) $\AA$. Les dimensions apparentes $\varepsilon_{\beta}$ (obs) et vraies $\tau(\mathrm{obs})$, ainsi que $\tau(\mathrm{cal})$ obtenues après affinement par la méthode moindrescarrés, sont indiquées sur la figure pour les directions cristallographiques considérées.

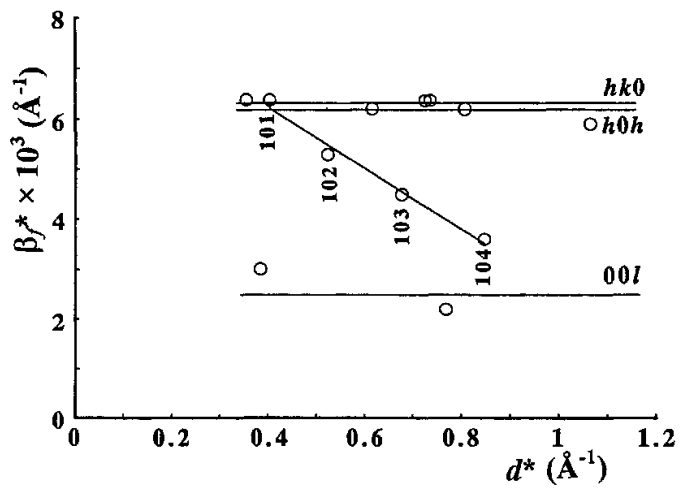

(a)

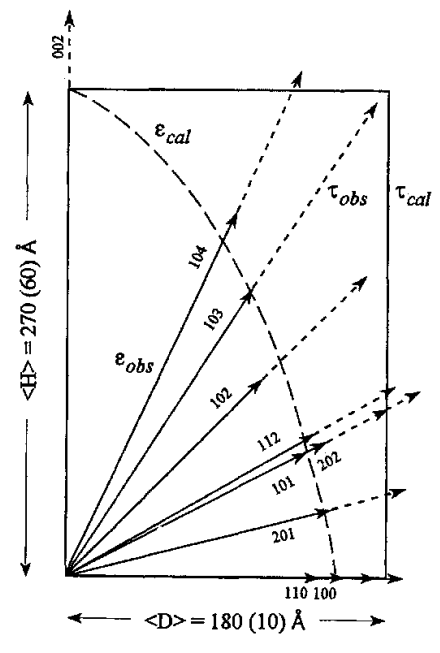

(b)

Figure 3: ZnO nanocristallin ex-hydroxynitrate. (a) Diagramme de Williamson-Hall; $(b)$ Section du cylindre moyen utilisé pour la modélisation de la forme des cristallites. $\longrightarrow$ : taille apparente observée $\varepsilon_{o b s ;}--\rightarrow$; taille vraie observée $\tau_{o b s}$. La courbe en pointillés $\varepsilon_{c a l}$ correspond au lieu géométrique des tailles apparentes calculées $[21,38]$. 


\subsection{Cristallites anisotropes avec des fautes: $\mathrm{ZnO}$ ex-oxalate}

Le diagramme de Williamson-Hall d'un échantillon de $\mathrm{ZnO}$ obtenu par décomposition thermique à $400^{\circ} \mathrm{C}$ de l'oxalate est représenté sur la figure $4[21,22]$. La dispersion des valeurs de $\beta_{f}{ }^{*} s^{\prime}$ explique par la présence de trois groupes de réflexions, dont les indices $h k l$ répondent aux conditions des équations (24). Il s'agit donc d'un exemple classique d'élargissement de raies de diffraction par des fautes d'empilement dans un solide de symétrie hexagonale. Les réflexions du groupe 1 sont peu dispersées et, donc, l'ordonnée à l'origine permet de suggérer des dimensions voisines dans les directions perpendiculaires aux plans $(h 00)$ et $(00 l)$. Une analyse moindres-carrés, fondée sur les équations (18) pour un modèle cylindrique a conduit au diamètre $\langle\mathrm{D}\rangle=402(9) \AA$ et à la hauteur $\langle\mathrm{H}\rangle=336(12) \AA$. La probabilité d'apparition d'une faute a été calculée pour les deux groupes de réflexions concernées (équ. 24) et a conduit à une probabilité moyenne $\alpha$ de 0,008 , ce qui correspond à une distance moyenne entre fautes de $316 \AA$, soit en moyenne une faute par cristallite.

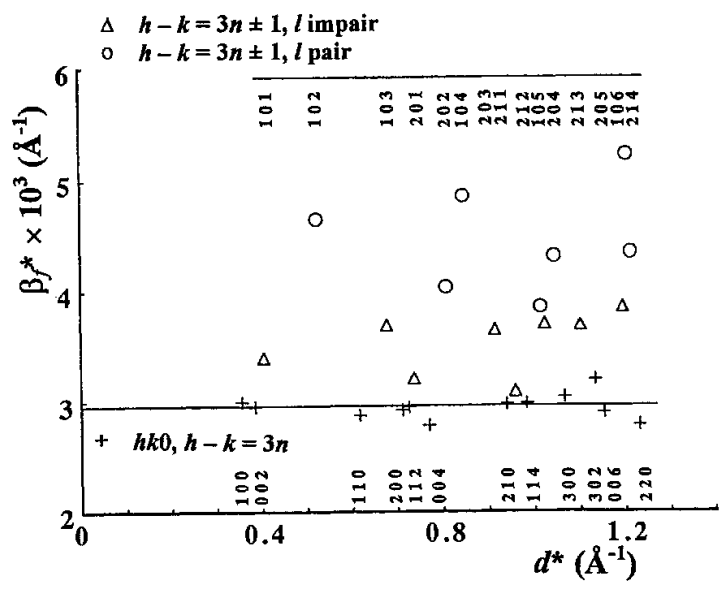

Figure 4: Diagramme de Williamson-Hall de ZnO nanocristallin ex-oxalate [22], montrant les trois groupes de réflexions définis par des conditions spécifiques sur les indices $h k l$.

\subsection{Cristallites anisotropes avec des distorsions: $\mathrm{Ni}(\mathrm{OH})_{2}$}

Le diagramme de Williamson-Hall d'un échantillon de $\mathrm{Ni}(\mathrm{OH})_{2}$ [39], tracé à partir de 24 réflexions, est représenté sur la figure 5 . Pour les réflexions $00 l, h 0 h$ et $h 00$ on observe des droites de pente positive qui indiquent la présence de microdistorsions dans le solide. L'ordonnée à l'origine non nulle pour les trois familles considérées montre un effet significatif de la petite taille des cristallites. Par ailleurs, la séquence $\beta^{*}(h 00, h k 0)<\beta^{*}(h 0 h)<\beta^{*}(00 l)$ $\mathrm{s}^{\prime}$ interprète à partir de cristallites possédant une forme de disque, l'axe $c$ de la maille hexagonale coïncidant avec l'axe du disque. Le comportement des largeurs intégrales des groupes de réflexions $(10 l, 11 l),(20 l, 21 l)$ et $30 l$ s'accorde parfaitement avec ce modèle. La présence de microcontraintes rend toutefois plus délicate l'évaluation précise des dimensions à partir de la largeur intégrale.

Les transformées de Fourier ont été calculées pour des réflexions d'ordres différents, $001 / 003 / 005,100 / 200$ et 101/202. Comme le montre la figure 6 , les courbes pour les réflexions $00 l$ ne sont pas superposées, ce qui confirme la présence de distorsions réticulaires. Le même comportement a été observé pour les autres familles. Les tailles apparentes calculées 
à partir de l'équation (19) sont $184 \AA, 545 \AA$ et $262 \AA$ dans les directions perpendiculaires à $(001),(100)$ et (101), respectivement. En supposant une forme cylindrique des cristallites, compatible avec la symétrie de $\mathrm{Ni}(\mathrm{OH})_{2}$, les paramètres $\mathrm{D}$ et $\mathrm{H}$ pour un cylindre moyen ont été déterminés à partir des tailles apparentes dans les directions perpendiculaires aux plans $(h 00)$ et $(00 l)$, soit $\langle\mathrm{D}\rangle=694 \AA$ et $\langle\mathrm{H}\rangle=184 \AA$. L'usage de l'équation (19) permet alors de calculer, dans la direction du vecteur diffractant $101\left(\phi_{z}=61,6^{\circ}\right)$, la taille apparente $\varepsilon_{\text {cal }}(101)$, soit 238 $\AA$, valeur qui est en accord satisfaisant avec la valeur observée, $262 \AA$. Ce résultat valide la morphologie en disque proposée pour les cristallites de l'échantillon de $\mathrm{Ni}(\mathrm{OH})_{2}$.

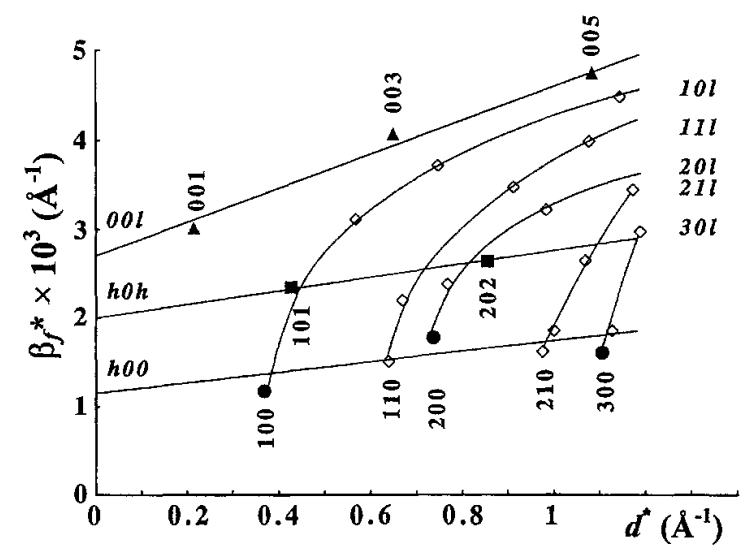

Figure 5: Diagramme de Williamson-Hall de $\mathrm{Ni}(\mathrm{OH})_{2}$ [39], montrant le comportement de plusieurs familles de raies de diffraction.

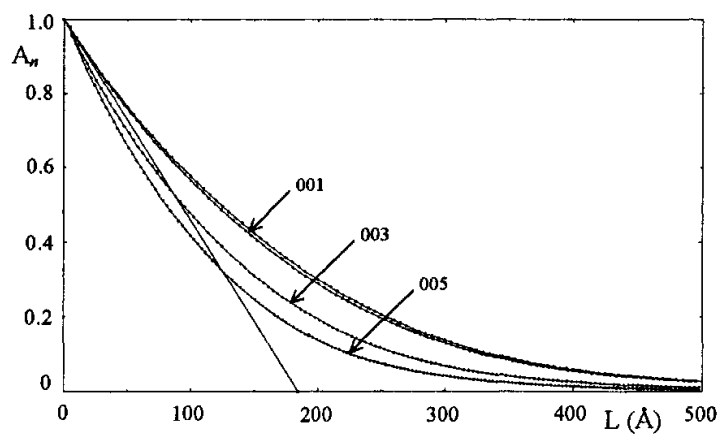

Figure 6: Coefficients de Fourier $A_{n}$ en fonction de $L$ pour les réflexions $00 l$ de $\mathrm{Ni}(\mathrm{OH})_{2}[39]$.

\subsection{Cristallites isotropes avec des dislocations: $\mathrm{CeO}_{2}$ ex-ammonium-nitrate}

Le diagramme de Williamson-Hall pour un échantillon de $\mathrm{CeO}_{2}$ obtenu par traitement thermique entre $550^{\circ} \mathrm{C}$ et $700^{\circ} \mathrm{C}$ de cérine hydratée, préparée elle-même à partir d'une solution de nitrate d'ammonium et de cérium, a révélé un élargissement anisotrope des raies de diffraction, due la présence de distorsions réticulaires anisotropes (voir figure $4 \mathrm{~b}$ dans la réf. 36). Cette anisotropie se traduit, entre autres, par une largeur de la réflexion 111 supérieure à celle de la raie 200 , ce qui est en accord avec une anisotropie élastique inférieure à l'unité. Ces 
distorsions ont été interprétées par l'existence d'une densité de dislocations dans les cristallites [35], à partir des approches décrites dans les sections 5.3 et 3.3. Les diagrammes modifiés de Williamson-Hall (équ. 30) et de Warren-Averbach (équ. 23) sont représentés sur la figure 7. Les largeurs, interpolées par l'équation (30) (figure $7 a$ ), suivent alors une variation régulière. On remarquera que le facteur de contraste $C$ introduit une séquence de réflexions $(200,111$, $400, \ldots)$ différente de la séquence habituelle du système cubique $(111,200,220, \ldots)$. Par ailleurs, dans l'approche de Fourier l'ordonnée à l'origine et la pente de la variation linéaire des coefficients de Fourier (figure $7 b$ ) conduisent aux coefficients de taille $\mathrm{A}^{\mathrm{T}}(\mathrm{L})$ et à $\mathrm{X}(\mathrm{L})=$ $\left(\pi \mathrm{b}^{2} / 2\right) \mathrm{\rho L}^{2} \ln \left(R_{\mathrm{e}} / \mathrm{L}\right)$, respectivement. L'ordonnée à l'origine de la fonction $\mathrm{A}^{\mathrm{T}}(\mathrm{L})=\mathrm{f}(\mathrm{L})$ fournit la taille apparente de Fourier $\varepsilon_{\mathrm{F}}$ (équ. 14), c'est-à-dire $198 \AA$. Les pentes ont été analysées à partir de l'équation

$$
\mathrm{X}(\mathrm{L}) / \mathrm{L}^{2}=-\rho\left(\pi \mathrm{b}^{2} / 2\right) \ln R_{\mathrm{e}}+\rho\left(\pi \mathrm{b}^{2} / 2\right) \ln \mathrm{L} .
$$

Le graphe $\mathrm{X}(\mathrm{L}) / \mathrm{L}^{2}$ en fonction de $\ln \mathrm{L}$ conduit directement à la densité de dislocations et au rayon effectif de coupure des dislocations, c'est-à-dire dans ce cas une densité de dislocation de $3,610^{14} \mathrm{~m}^{-2}$. L'étude a montré que la densité de dislocations décroît lorsque la température s'élève pour devenir négligeable vers $700^{\circ} \mathrm{C}$.

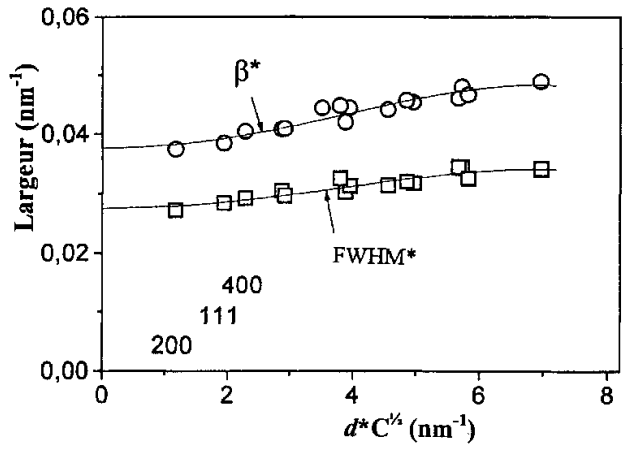

(a)

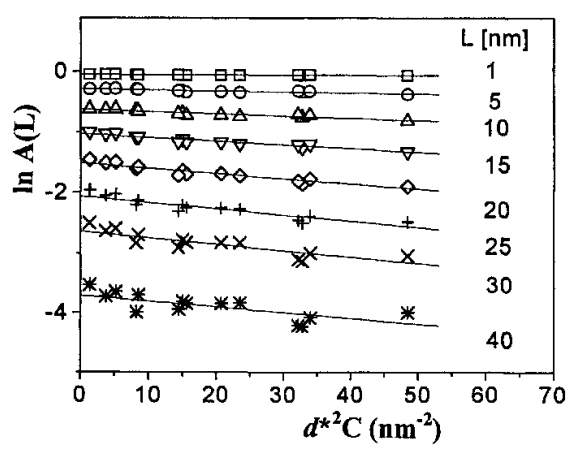

(b)

Figure 7: $\mathrm{CeO}_{2}$ nanocristallin ex-ammonium-nitrate. (a) Diagramme de Williamson-Hall modifié, selon l'équation (30); (b) Diagramme de Warren-Averbach modifié, selon l'équation (23) [35].

\section{REMARQUES FINALES}

Les défauts rencontrés dans les matériaux réels conduisent à des comportements variés des largeurs de profil des raies de diffraction des rayons X. L'identification des imperfections structurales à l'origine de ces élargissements (isotropes et anisotropes) nécessite une inspection attentive de la variation des largeurs en fonction de la variable réciproque $d^{*}$ et de leur dépendance avec la direction cristallographique (ordre des réflexions). Plusieurs approches, plus ou moins sophistiquées, permettent de procéder à leur analyse. L'approche simple des diagrammes de Williamson-Hall éclaire sur les défauts présents dans un solide, à condition toutefois que la correction instrumentale soit faite correctement, notamment par la méthode de Voigt si celle-ci est applicable. L'approche de Fourier est généralement considérée comme la plus rigoureuse. Dans tous les cas des données de haute qualité sont requises pour obtenir des 
renseignements précis, allant parfois jusqu'à une description tridimensionnelle des caractères microstructuraux. Les méthodes de modélisation repoussent les limites des applications en aidant à restituer des profils de raies de diffraction présentant un recouvrement. Cette approche commode et séduisante ne doit pas masquer le biais possible que peut entraîner l'usage de telle ou telle fonction mathématique (voir, par exemple, réf. 20). Néanmoins, ces outils demeurent incontournables dans l'analyse microstructurale à partir du profil des raies. Ceci se traduit par l'usage de logiciels variés utilisant la modélisation analytique des diagrammes de diffraction [par exemple, 37,40], l'usage de la méthode de Rietveld et de son option 'pattern matching' (voir, par exemple, l'application récente à des poudres d'oxyde d'yttrium nanocristallin [41]) et l'émergence d'une modélisation fondée sur l'usage des paramètres physiques à l'origine de l'élargissement des raies de diffraction [20,42]. Pour conclure, si l'essor considérable de la diffraction par les poudres au cours des dix dernières années repose pour beaucoup sur l'analyse structurale, il apparait clairement que la caractérisation microstructurale constitue un secteur qui va encore évoluer dans les années futures.

\section{Références}

[1] Langford J. I. et Louër D., Rep. Prog. Phys., 59 (1996) 131-234.

[2] 'Defect and microstructure analysis by diffraction', Ed. R. L. Snyder, J. Fiala \& H. J. Bunge (Oxford:IUCr/OUP, 1999).

[3] 'The Rietveld method', Ed. R. A. Young (Oxford: IUCr/OUP, 1995).

[4] Le Bail A., Accuracy in Powder Diffraction II, Ed. E. Prince \& J. K. Stalick (NIST Special Publ. 846, 1992) pp. 142-153.

[5] Delhez R., de Keijser T. H., Langford, J. I., Louër D., Mittemeijer E. J. et Sonneveld E. J., The Rietveld method, Ed. R. A. Young (Oxford: IUCr/OUP, 1995) pp. 132-166.

[6] Toraya H., J. Appl. Cryst. 18 (1985) 351-358.

[7] Thompson P., Cox D. E. et Hastings J. B., J. Appl. Cryst., 20 (1987) 79-83.

[8] Rodriguez-Carvajal J., Guide d'utilisation du programme FULLPROF (2001) (FULLPROF est disponible à http://www-llb.cea.fr/fullweb/powder.htm).

[9] Guinier A., 'Théorie et technique de la radiocristallographie' (Dunod, Paris, 1956).

[10] Wilson A. J. C., 'X-ray optics' (Methuen, London, 1962).

[11]Bertaut E. F., Acta Cryst., 3 (1950) 14-18.

[12] Warren B. E., 'X-ray Diffraction' (Reading MA: Addison-Wesley, 1969).

[13]Delhez R. and Mittemeijer E. J., J. Appl. Cryst., 9 (1976) 233-234.

[14] Wagner C. N. J., Local atomic arrangements studied by X-ray diffraction, Ed. J. B. Cohen \& J. E. Hilliard (Gordon \& Breach, Sci. Pub.: NY, 1966) pp. 219-269.

[15] Stokes A. R. et Wilson A. J. C., Proc. Phys. Soc. Lond., 56 (1944) 174-181.

[16]Langford J. I., Accuracy in Powder Diffraction, Ed. E. Prince \& J. K. Stalick (NIST Special Publ. 846, 1992) pp. 110-126.

[17]Langford J. I., Delhez R., de Keijser Th. H. et Mittemeijer E. J., Aust. J. Phys., 41 (1988) 173-187.

[18]Langford J. I. et Louër D., J. Appl. Cryst., 15 (1982) 20-26.

[19] Vargas R., Louër D. et Langford J. I., J. Appl. Cryst., 16 (1983) 512-518.

[20]Langford J. I., Louër D. et Scardi P., J. Appl. Cryst., 33 (2000) 964-974.

[21]Langford J. I., Boultif A., Auffrédic J.-P. et Louër D., J. Appl. Cryst., 26 (1993) 22-33.

[22] Auffrédic J.-P., Boultif A., Langford J. I. et Louër D., J. Am. Ceram. Soc., 78 (1995) 323-328.

[23] Toraya H., Powder Diffr., 4 (1989) 130-136. 
[24] Krivoglaz M. A., 'Theory of X-ray and thermal neutron scattering by real crystals' (New York: Plenum Press, 1969).

[25] Wilkens M., Phys. Stat. Sol. (a), 2 (1970) 359-370.

[26]Ungár T. et Tichy G., Phys. Stat. Sol. (a), 171 (1999) 425-434.

[27] Klimanek P. et Kužel R., J. Appl. Cryst., 21 (1988) 59-66.

[28] Ungár T., Dragomir I., Révész Á. et Borbély A., J. Appl. Cryst., 32 (1999) 992-1002.

[29] Wilson A. J. C., 'Mathematical theory of X-ray powder diffractometry' (Eindhoven: Philips, 1963).

[30]Louër D. et Langford J. I., J. Appl. Cryst., 21 (1988) 430-437.

[31] Stokes A. R., Proc. Phys. Soc., 61 (1948) 382-391.

[32]Langford J. I., J. Appl. Cryst., 11 (1978) 10-14.

[33] Williamson G. K. et Hall W. H., Acta Metall., 1 (1953) 22-31.

[34]Ungár T. et Borbély A., Appl. Phys. Lett., 69 (1996) 3173-3175.

[35] Ungár T., Dragomir-Cernatescu I., Louër D. et Audebrand N., J. Phys. Chem. Sol., 62 (2001) 1935-1941.

[36] Audebrand N., Auffrédic J.-P. et Louër D., Chem. Mater., 12 (2000) 1791-1799.

[37] Louër D. et Audebrand N., Adv. X-ray Anal., CD-ROM, Newtown Square: ICDD, Vol. 41 (1999) 556-565.

[38] Louër D., Auffrédic J.-P., Langford J. I., Ciosmak D. et Niepce J. C., J. Appl. Cryst., 16 (1983)183-191.

[39] Audebrand N. et Louër D., J. Phys. IV France, 8 (1988) 109-118.

[40]Dong Y. H. et Scardi P., J. Appl. Cryst., 33 (2000) 184-189.

[41] Louër D., Bataille T., Roisnel T. et Rodriguez-Carvajal J., Powder Diffr. (2003) in press.

[42] Scardi P, Dong Y. H. et Leoni M., Mater. Sci. Forum, 378-381 (2001) 132-141. 